Cochrane Database of Systematic Reviews

\title{
Trunk training for improving activities in people with stroke (Protocol)
}

Thijs L, Denissen S, Mehrholz J, Elsner B, Lemmens R, Verheyden GSAF

Thijs L, Denissen S, Mehrholz J, Elsner B, Lemmens R, Verheyden GSAF.

Trunk training for improving activities in people with stroke (Protocol).

Cochrane Database of Systematic Reviews 2020, Issue 9. Art. No.: CD013712.

DOI: 10.1002/14651858.CD013712.

www.cochranelibrary.com

Trunk training for improving activities in people with stroke (Protocol) 
TABLE OF CONTENTS

HEADER 1

ABSTRACT

BACKGROUND

OBJECTIVES

METHODS

ACKNOWLEDGEMENTS

REFERENCES

ADDITIONAL TABLES

APPENDICES

HISTORY

CONTRIBUTIONS OF AUTHORS

DECLARATIONS OF INTEREST

SOURCES OF SUPPORT 
[Intervention Protocol]

\section{Trunk training for improving activities in people with stroke}

Liselot Thijs ${ }^{1}$, Stijn Denissen 2,3 , Jan Mehrholz ${ }^{4}$, Bernhard Elsner ${ }^{5}$, Robin Lemmens ${ }^{6}$, Geert SAF Verheyden ${ }^{1}$

1Department of Rehabilitation Sciences, KU Leuven, Leuven, Belgium. 2AIMS lab, Center for Neurosciences, UZ Brussel, Vrije Universiteit Brussel, Brussels, Belgium. ${ }^{3 i c o m e t r i x, ~ L e u v e n, ~ B e l g i u m . ~}{ }^{4}$ Department of Public Health, Dresden Medical School, Technical University Dresden, Dresden, Germany. ${ }^{5}$ Department of Public Health, Dresden Medical School, Technical University Dresden, Dresden, Germany. ${ }^{6}$ Department of Neurosciences, Laboratory for Neurobiology, UZ Leuven, Leuven, Belgium

Contact address: Geert SAF Verheyden, geert.verheyden@faber.kuleuven.be.

Editorial group: Cochrane Stroke Group.

Publication status and date: New, published in Issue 9, 2020.

Citation: Thijs L, Denissen S, Mehrholz J, Elsner B, Lemmens R, Verheyden GSAF. Trunk training for improving activities in people with stroke (Protocol). Cochrane Database of Systematic Reviews 2020, Issue 9. Art. No.: CD013712. DOI: 10.1002/14651858.CD013712.

Copyright @ 2020 The Cochrane Collaboration. Published by John Wiley \& Sons, Ltd.

\section{A B S T R A C T}

\section{Objectives}

This is a protocol for a Cochrane Review (intervention). The objectives are as follows:

To determine the effectiveness of trunk training after stroke in comparison to no therapy or to control therapy, either dose-matched or not. The review will assign the outcome measures to the levels defined in the ICF model and the review will evaluate the effect of different types of trunk training. 


\section{B A C K G R O U N D}

\section{Description of the condition}

Stroke can be a devastating condition and, although progress has been made in its understanding and treatment, it is still the second leading cause of death worldwide and the second most common cause of disability-adjusted life-years (GBD 2019). Stroke can affect vision, cognition, communication, and sensorimotor function. Even within this last domain, stroke can induce a wide range of deficits, from none or only very minor deficits to a complete paralysis of the affected side of the body.

One frequently-observed motor consequence is trunk impairment, which contributes to sitting balance deficits (Lee 2015; Verheyden 2004). This is particularly seen in the early stages after a stroke, but can also occur in the later stages,

Adequate trunk function is the result of different core components, including balance, muscle function, and position sense. A stroke can have an impact on one or more of these components.

Early after stroke, researchers observed reduced stability and a greater sway during dual tasks (Harley 2006). While performing the forward reach task, displacement of the centre of pressure was shown to be decreased in people who have had a stroke (Messier 2004). When people who had been severely affected by stroke reached forward in an upright-seated position, the erector spinae muscles on the paretic side showed significantly higher activity than on the non-paretic side. On the other hand, the rectus abdominis muscles on the paretic side were found to be significantly less active than on the non-paretic side (Dickstein 1999). This is assumed to be related to the reduced trunk muscle strength reported previously (Bohannon 1992). Later in the rehabilitation process, trunk weakness is still detectable (Quintino 2018). Lee and colleagues reported that the abdominal muscles were significantly thinner on the affected side, and that the ratio between the thickness during rest and contraction of the abdominal muscles was significantly lower on the affected side. A higher value of this latter ratio related to better trunk function (Lee 2018). Compared to healthy age-matched people, people who had experienced a stroke had alternated position sense of the trunk after a forward moving task in the sagittal and transverse plane (Ryerson 2008).

Impaired trunk components have a negative impact on trunk function. Trunk function provides the ability to sit and remain upright against gravity. It also ensures moving trunk and body freely, adopting different sitting positions, and performing seated reach without losing balance. Furthermore, adequate trunk function is a key requirement for the upper and lower part of the trunk to move separately from each other, for instance whilst walking (Davis 1990; Karthikbabu 2011; Verheyden 2004).

Clinically, rehabilitation of trunk function is a key milestone in recovery, meaning that people after stroke should be able to sit unsupported on a bed or plinth with their trunk and head in an upright position (Smith 1999). Both trunk function and sitting balance are strongly correlated with functional independence (Di Monaco 2010; Santos 2019; Verheyden 2006), and are significant and independent predictors of motor and functional outcome (Hsieh 2002; Verheyden 2007). Regaining trunk function with trunk training is therefore warranted, and the importance of trunk training is recognised in the literature (Alhwoaimel 2018; Bank 2016; Cabanas-Valdés 2013; Sorinola 2014; Souza 2019; Van Criekinge 2019). Because it is an important milestone in rehabilitation, trunk training receives most attention in the acute and early rehabilitation phase (Smith 1999). Yet even at a later phase, there can still be considerable impairment in trunk function (Lee 2015; Verheyden 2004). Thus, trunk training may be beneficial for people in all phases after stroke.

\section{Description of the intervention}

Trunk training aims to enhance trunk muscle strength and trunk muscle endurance, thereby providing a stable base for selective and coordinated movements of (part of) the trunk, the head, or extremities. The specific approach can vary in the different rehabilitation phases.

Early after stroke, trunk training might be undertaken in a lying or sitting position. Objectives of training are to increase trunk and body muscle activation during transfers, and to improve an upright and aligned position, stimulating dynamic sitting balance. The latter will result in adequate weight shifts and the ability to reach with the upper limb within the limits of stability. Improved coordination results in better selective movements of the shoulder and pelvic girdle. If basic transfers, reaching and sitting balance are achieved, therapy goals will shift towards improving muscle strength, achieving a wider range of movements in sitting, including more dynamic ability and improved dual tasks, which are required for activities of daily living.

The literature describes a diverse range of trunk training approaches (Alhwoaimel 2018; Bank 2016; Cabanas-Valdés 2013; Sorinola 2014; Souza 2019; Van Criekinge 2019). Seven broad approaches can be distinguished: 1) core stability training; 2) electrostimulation; 3) selective trunk training; 4) sitting reaching training; 5) static inclined surface training; 6) unstable surface training; and 7) weight-shift training.

Core stability training is the isometric strengthening of the trunk muscles, i.e. the musculature of the pelvic and hip girdle, lumbar, abdominal, cervical and periscapular muscles (e.g. Yoo 2010). Electrostimulation targets one or more of these core muscles with electrophysiological stimulation (e.g. Ko 2016). Selective trunk training aims to improve selective movements of the upper (shoulder girdle) and lower part (pelvic girdle) of the trunk, through voluntary trunk activation in different directions (e.g. An 2017). Training by use of sitting reaching therapy focuses on improving sitting balance by reaching beyond arm's length with the nonaffected hand, in different directions (e.g. Ada 2006). During static inclined surface training, the person remains on a fixed, static inclined surface while performing voluntary trunk activation (e.g. Fujino 2015). Unstable surface therapy is the therapeutic approach of training voluntary trunk activation on an unstable or moving surface that causes constant perturbations, for example on a physioball or a mechanical device (e.g. Karthikbabu 2011a). Finally, weight shift training involves shifting the body weight in a single direction to the limits of sitting ability (e.g. Jung 2016).

A summary of evidence is needed to inform clinical practice, providing both an overview of the effects of trunk training and an assessment of the individual types of trunk training. 


\section{How the intervention might work}

The trunk is the core of the body. It has a key role in stabilising the body during movements of the head and extremities, and provides support during sitting balance (Houglum 2012; Wee 2015). After a stroke, trunk muscle strength is reduced compared to that of healthy controls (Silva 2015; Tanaka 1998), leading to impaired trunk function and sitting balance. Trunk training focuses on improving trunk and core muscle strength to improve sitting balance, which is advantageous for enhancing basic activities of daily living.

In a cross-sectional study, we observed a relationship between decreased trunk function and poor standing balance, mobility, and functional ability (Verheyden 2006). Furthermore, initial trunk function is a predictor of functional performance (Duarte 2002; Hsieh 2002; Verheyden 2007). Trunk training would improve trunk function, but could also positively influence other components of the International classification of functioning (ICF) framework (WHO 2001), such as mobility, balance, functional outcome, and potentially quality of life after stroke.

Trunk training may increase the size and strength of trunk muscles, and this could have a positive influence on trunk muscle endurance. This may improve sitting balance, evolving from adequate static sitting balance to appropriate dynamic sitting balance and refining of trunk coordination with increased limits of stability, resulting in a positive effect on activities of daily living (such as washing and selfcare).

Due to the association between trunk function, standing balance and mobility (Duarte 2002; Hsieh 2002; Verheyden 2006; Verheyden 2007), an improvement in trunk function could positively impact activities such as walking up the stairs or taking a shower or bath, and so have a positive effect on activities of daily living.

\section{Why it is important to do this review}

So far, we have identified five literature reviews investigating the effect of trunk training. An overview of the published reviews is presented in Table 1. Cabanas-Valdés and colleagues included 11 trials in their review. They did not perform a meta-analysis, but summarised that trunk training had a moderate positive effect on trunk function (Cabanas-Valdés 2013). Sorinola 2014 included six trials, conducted a meta-analysis, and concluded that sitting balance and trunk training had no effect on trunk function. In contrast, Alhwoaimel 2018, who included 17 trials, performed a meta-analysis that showed a large effect of trunk training on trunk function. A recent review combined 22 trials and also noted a large effect on trunk outcome (Van Criekinge 2019). The most recent review assessed the effect of trunk training in the first three months after stroke. The authors included nine trials and found a significant effect of trunk training on trunk outcome (Souza 2019).

All reviews investigated the effect of training on trunk function. Four reviews included outcome measures other than trunk function, such as standing balance, gait, and functional performance (Cabanas-Valdés 2013; Sorinola 2014; Souza 2019; Van Criekinge 2019). By using different search strategies and analyses, all concluded that trunk training had a positive effect on balance, varying from a small to a huge effect. However, some reviews were based on a limited number of trials. Therefore, caution in generalising this conclusion is still necessary. The aim of one review was to examine the effect of trunk training on arm-hand performance, but no studies were identified (Alhwoaimel 2018). Sorinola 2014 investigated the effect of trunk training on functional performance, and included two trials with a total of 42 participants. They reported that trunk function was not effective for improving functional outcome, measured by the Functional Independence Measure scale. Trunk training is a fast-growing field of research. We undertook a preliminary search and identified 29 trunk training trials, warranting a comprehensive synthesis of the literature.

It is clear that interest in poststroke trunk rehabilitation is growing, given that the number of studies is high and increasing. Often, the same outcome parameters are examined, such as trunk function, standing balance, and gait. Only two reviews conducted metaanalyses to evaluate the effect of trunk training on other outcome parameters (Alhwoaimel 2018; Sorinola 2014). No other recent review adopted a meta-analytic approach to examine whether trunk training could positively improve activities of daily living. The majority of reviews only described the type of trunk therapy. Only one review examined the effect of distinct trunk training approaches, and this only gave a descriptive summary of review results (Cabanas-Valdés 2013).

An important element in all the reviews is that they combined trials where the control groups received no therapy, only conventional therapy (usual care) or conventional therapy with additional therapy. In the experimental group, participants received an additional intervention on top of conventional therapy. Combining these different types of control therapy should be avoided, and the literature should answer whether trunk training has an effect when comparing it to either no therapy or other therapy. Apparently, no review has yet answered these important questions.

Thus, the need for this Cochrane Review is high. It will make available and synthesise all the information about the effect of trunk training poststroke: of the different key outcomes included in the trials, evidence concerning the different types of trunk training, training in the different phases poststroke, and trunk training compared to no therapy and to other therapy. Finally, a Cochrane Review will also be kept up to date, assuring permanent state-ofthe-art in this intensively-studied research field.

\section{O B JECTIVES}

To determine the effectiveness of trunk training after stroke in comparison to no therapy or to control therapy, either dosematched or not. The review will assign the outcome measures to the levels defined in the ICF model and the review will evaluate the effect of different types of trunk training.

\section{METHODS}

\section{Criteria for considering studies for this review}

\section{Types of studies}

We will include randomised controlled trials (RCTs) only.

\section{Types of participants}

Adult participants (18 years or older) with either ischaemic or haemorrhagic stroke. We will exclude trials that include other diseases in addition to stroke, unless they report separate results for the participants of interest. If the trials report the effect of trunk 
training separately by pathology, we will consider that trial for our analysis.

\section{Types of interventions}

We will include trials that compare any type of trunk training (experimental group) against no therapy, or against either nondose-matched or dose-matched control therapy (control group). The interventions can be undertaken in isolation or in addition to conventional therapy, as long as both groups receive this. To improve the certainty that the effects that we will evaluate in this review can be attributed to trunk training, we will only include trials in which the trunk is trained specifically, as described in the types of trunk training in the Background. If trunk training is embedded in a broader training concept, such as circuit training or a general strength programme, we will not include that study in this review.

Our primary interest is trunk training provided in a seated or lying position. The participants can be positioned on a stable or unstable surface, and can be lying in a supine, crooked, or sideways position. We expect a wide variability in types of trunk training interventions. To give a clear overview, we will report on the following different types of trunk training:

- core stability training;

- electrostimulation;

- selective trunk training;

- sitting reaching training;

- static inclined surface training;

- unstable surface training;

- weight-shift training; and

- other types of training.

We will also include studies that aim to improve trunk function where the intervention is performed in a standing position, but only if the study also includes trunk training in a seated or lying position, and if this forms the majority of the therapy delivered. Since we will only investigate the effect of trunk training, we will not include pharmacological or surgical interventions.

\section{Types of outcome measures}

We expect that the RCTs will use different instruments to evaluate the outcome measures of interest. We will extract data if the trials report the outcome using the below-listed scales, or if they report the data using a comparable rating scale. We will assign the outcome measures to the levels defined in the ICF model (WHO 2001).

We will examine if trunk training has an effect on:

- the level of body function, including: trunk function, leg and arm-hand function, and standing balance;

- the level of activity and participation, including: activities of daily living, arm-hand activity, walking ability, and quality of life;

- death and serious adverse events, including falls.

The primary outcome will be activities of daily living (ADL), whereas the secondary outcomes aim to assess body function, activity and participation level, and adverse events. We will extract data for our primary and secondary outcome measures immediately after the intervention.

\section{Primary outcomes}

- Activities of daily living: measured by the Barthel index (Mahoney 1965), or modified Barthel index (Collin 1988). This scale is the priority scale for data extraction, after which we will consider the Functional Independence Measure (FIM) (Keith 1987), the reintegration in normal living index (Wood-Dauphinee 1988), and other comparable outcome parameters.

\section{Secondary outcomes}

- Trunk function: if the trial measured trunk function, sitting balance, or both using the Trunk Impairment Scale (TIS) (Verheyden 2004), we will extract these results as the priority scale, followed by data on the Trunk Control Test (Collin 1990), and forward reach (Duncan 1990), or a comparable measure.

- Arm-hand function: we will give priority to the Fugl-Meyer Assessment (upper extremity) (Fugl-Meyer 1975), or use data from a corresponding measure if the trial does not report the measure of choice.

- Arm-hand activity: we will prioritise data from the Action Research Arm Test (ARAT) (Lyle 1981), followed by the upper limb Chedoke-McMaster Stroke Assessment (Moreland 1993), or a comparable measure.

- Standing balance: we will extract data from the Berg Balance Scale as our measure of choice (Berg 1992), or the balance part of the Tinetti Scale (Tinetti 1986), or a comparable measure.

- Leg function: we will give priority to data from the Fugl-Meyer Assessment (lower extremity) (Fugl-Meyer 1975), or include data from a comparable measure.

- Walking ability: first we will look at whether data are available that evaluate walking speed. Priority goes to data measured with the 10-Meter Timed Walk Test (Collen 1990), followed by data from the Timed Up and Go Test (Mathias 1986), or a comparable measure. If the trial has not undertaken a gait speed evaluation, we will extract data from other scales, such as (but not limited to) the gait part of the Tinetti Scale (Tinetti 1986), or Functional Ambulation Categories (Holden 1984).

- Quality of life: we will collect data from the Stroke Impact Scale as our priority scale (Duncan 1999), or use similar quality of life outcomes.

- Reporting of death and serious adverse events, including falls.

We expect that the included trials might report a variability of other outcome measures. Therefore, we will list all other outcome measures of the included trials in table format.

\section{Search methods for identification of studies}

See the methods for the Cochrane Stroke Group 'Specialised register'. We will search for trials in all languages and arrange for the translation of relevant articles where necessary.

\section{Electronic searches}

We will search the Cochrane Stroke Group trials register and the following electronic databases.

- Cochrane Central Register of Controlled Trials (CENTRAL; latest issue) in the Cochrane Library

- MEDLINE Ovid (from 1946) (Appendix 1)

- Embase Ovid (from 1974) 
- CINAHL (Cumulative Index to Nursing and Allied Health Literature) (from 1982)

- PEDro (from 1900)

- Scopus (from 1996)

- SPORTDiscus EBSCO (from 1982)

- ProQuest Dissertations and Theses (from 1997)

We will model the search strategies for databases on the search strategy designed for MEDLINE (Appendix 1), in consultation with the Cochrane Stroke Group's Information Specialist. We will combine all search strategies deployed with subject strategy adaptations of the sensitive search strategy designed by Cochrane for identifying randomised controlled trials, as described in the Cochrane Handbook for Systematic Reviews of Interventions (Lefebvre 2019).

We will search the following ongoing trials registers:

- US National Institutes of Health Ongoing Trials Register ClinicalTrials.gov (www.clinicaltrials.gov/);

- World Health Organization (WHO) International Clinical Trials Registry Platform (who.int/ictrp/en/).

\section{Searching other resources}

In an effort to identify further published, unpublished, and ongoing trials, we will:

- handsearch the bibliographies of included studies and any relevant systematic reviews identified for further references to relevant trials, and use Google Scholar to forward track relevant references (scholar.google.co.uk/);

- contact original authors for clarification and further data if trial reports are unclear;

- where necessary, contact experts/trialists/organisations in the field to obtain additional information on relevant trials;

- conduct a search of various grey literature sources using the Canadian Agency for Drugs and Technologies in Health (CADTH) Grey Matters checklist (www.cadth.ca/resources/ finding-evidence/grey-matters) (from 1989). Additionally, we will search the ProQuest Dissertations and Theses database (from 1997).

\section{Data collection and analysis}

\section{Selection of studies}

Two review authors (LT and SD) will independently screen titles and abstracts of the references obtained as a result of our searching activities, and will exclude obviously irrelevant reports. We will retrieve the full-text articles for the remaining references. Two review authors (LT and SD) will independently screen these fulltext articles, identify studies for inclusion, and identify and record reasons for exclusion of the ineligible studies. We will resolve any disagreements through discussion or, if required, we will consult a third person (GV). We will collate multiple reports of the same study so that each study, not each reference, is the unit of interest in the review. We will record the selection process and complete a PRISMA flow diagram (Liberati 2009). We will use Covidence for text screening and de-duplication of the citations (Covidence 2017).

\section{Data extraction and management}

Two review authors (LT and SD) will independently extract data from included studies using an extraction form. We will obtain information about trial publication and participants, eligibility criteria, intervention, and results from both the experimental and control group. We will use Covidence for data extraction (Covidence 2017).

We will extract data for our primary and secondary outcomes. We will only include trials which investigate stroke and other pathologies if they provide outcome data separately for people with stroke.

We will collect the following information about the trials' participants:

- mean age and standard deviation (SD);

- number of participants;

- sex;

- type and location of the stroke event;

- stroke severity at baseline, by means of the National Institutes of Health Stroke Scale (NIHSS) or comparable scale;

- hyper-acute treatment of stroke;

- presence of other stroke-related impairments, such as aphasia, neglect or hemianopia;

- comorbidity at baseline; and

- mean time and SD after stroke in days, weeks, or months at the start of the intervention.

We will record whether studies are multicentre, and collect information on their geographical location and setting.

To evaluate the effect of different types of trunk training on trunk function, we will divide the trunk training intervention into eight categories. Two independent investigators (LT and SD) will review the intervention for each study and classify it into the appropriate type of training. A third author (GV) will review this if there is any disagreement. If an included study combines two or more types of training in the experimental intervention, we will classify it based on the type of training that forms the largest component.

We will collect the following details of the interventions:

- type of intervention;

- length of intervention in minutes, days, or weeks;

- total number of repetitions in the experimental and control group;

- total minutes of intervention in the experimental and control group; and

- total minutes of conventional therapy in both groups.

We will use mean time since stroke plus the intervention period to classify trials according to poststroke phase:

- (hyper) acute: from within the first 24 hours up to seven days;

- early subacute: from more than seven days up to three months;

- late subacute: more than three months up to six months; and

- chronic phase: more than six months poststroke. 
The mean time poststroke and the period of intervention must be within one of the above-mentioned phases if we are to consider the study for analysis by phase after stroke (Bernhardt 2017).

To enhance transparency, we will use the TIDieR (Template for Intervention Description and Replication) checklist for each included intervention, to provide details of the experimental therapy (Hoffmann 2014).

We will present all outcome data in additional tables for both the intervention and control group.

\section{Assessment of risk of bias in included studies}

Two review authors (LT and SD) will independently assess the risk of bias for each study, using the criteria outlined in the Cochrane Handbook for Systematic Reviews of Interventions (Higgins 2011). We will resolve any disagreements by discussion or by involving a third review author (GV). We will assess the risk of bias according to the Cochrane 'Risk of bias' tool for randomised trials, evaluating the following domains.

- Random sequence generation

- Allocation concealment

- Blinding of participants and personnel

- Blinding of outcome assessment

- Incomplete outcome data

- Selective outcome reporting

- Other bias

We will grade the risk of bias for each domain as high, low, or unclear. We will include a justification along with relevant information from the study report in the 'Risk of bias' tables.

Review authors will not evaluate the risk of bias for studies in which they participated as an author.

\section{Measures of treatment effect}

For dichotomous data, we will calculate and report odd ratios (ORs) with $95 \%$ confidence intervals (Cls). For continuous outcomes, we will calculate standardised mean differences (SMDs) with 95\% Cls (if studies measure the same outcome using different tools), or use mean differences (MDs) and 95\% Cls when all studies used the same measurement tool. MDs provide more clinically relevant information, so we will conduct a separate analysis to combine data for any outcomes where more than six trials used the same specific outcome measure, and display results as MD with $95 \% \mathrm{Cls}$. To ensure that meta-analysis is clinical meaningful, we will only combine trials when participants, interventions, and outcomes are judged to be sufficiently similar. If trials are not sufficient similar a narrative summary of the trial will be included.

We will extract or calculate mean change score and standard deviations (SDs) from the pre- and postintervention time point for each available outcome measure. If a study provides the data as median and interquartile range, we will convert the data to mean change score and SD for large studies (with more than 100 participants in each group). For trials with smaller sample sizes, we will not consider median and interquartile range data further, under the hypothesis that data are skewed and not normally distributed (Higgins 2019a; Wan 2014).

\section{Unit of analysis issues}

We will consider two unit of analysis issues in this review: clusterrandomisation, and inclusion of trials with multiple intervention arms.

We will consider the inclusion of a cluster-randomised trial; however, we will apply the methods recommended in the Cochrane Handbook for Systematic Reviews of Interventions (Higgins 2019c).

If trials include multiple interventions, we will only include the results if the trial presents data of the different interventions that are relevant to this review separately. To avoid double counts, we will not include a study with multiple interventions in the same forest plot. If both interventions are relevant, we will pool the groups by combining the means and SDs, as recommended in the Cochrane Handbook for Systematic Reviews of Interventions (Higgins 2019a). We will apply the same approach when a trial compares the same type of intervention but with a different training intensity.

\section{Dealing with missing data}

We will contact study authors to acquire missing data.

When a study presents mean change scores before and after the intervention but does not report the SDs, we will first contact the authors of the RCT to request the missing data. If we receive no response, we will calculate the SDs using the pooled correlation coefficient (Higgins 2019a).

\section{Assessment of heterogeneity}

We will calculate the $\mathrm{I}^{2}$ statistic to measure heterogeneity among the trials in each analysis (Higgins 2003). We will consider an $\mathrm{I}^{2}>$ $75 \%$ as a considerable level of heterogeneity. In such a scenario, we will explore the potential sources of heterogeneity (Deeks 2019).

\section{Assessment of reporting biases}

We will avoid reporting bias primarily by using an extensive search strategy of databases and handsearching of reference lists. Furthermore, we will evaluate reporting bias for the outcome measures where we include more than 10 trials by visual inspection of funnel plots. In case doubt remains, and if more than 10 trials are included, we will conduct Eggers' Regression Test for funnel plot asymmetry $(\mathrm{P}<0.05)$ (Sterne 2005).

\section{Data synthesis}

We will pool the results of all eligible studies to present an overall estimate of the effect of trunk training on all outcome measures and according to type of training and phase after stroke, where possible. We will split the meta-analyses for each outcome. A first analysis will include studies investigating the effect of (additional) experimental training versus no control training. A second analysis will investigate the effect of (additional) experimental training versus non-dose-matched and dose-matched (additional) control training. We will perform a meta-analysis for each type of trunk training if we include two or more trials for that type of training.

We will perform statistical analyses within Cochrane's Review Manager software RevMan Web. We will apply fixed-effects models for continuous outcome and a random-effects model for dichotomous data. 


\section{Subgroup analysis and investigation of heterogeneity}

If heterogeneity is high ( $12>75 \%$ ), we will conduct a subgroup analysis and perform a meta-regression, if possible.

We will undertake a subgroup analysis for the different phases after stroke. We will only consider a subgroup analysis if we include at least six studies for continuous data and four for categorical data (Fu 2010). We will group outcomes according to the phases indicated earlier:

- (hyper) acute: from within the first 24 hours up to seven days;

- early subacute: from more than seven days up to three months;

- late subacute: from more than three months up to six months; and

- chronic phase: from more than six months poststroke.

We will use the test for subgroup differences to evaluate whether the two subgroups differ significantly from each other $(P<0.05)$.

We will assess the influence of the different potential effect moderators (explanatory variables). These variables may have an influence on the effect size of the intervention. We will calculate these influences of moderators using a meta-regression analysis using the "Metafor" package in R (R; Viechtbauer 2010a). The potential moderators (if available) are:

- study quality;

- age of participants;

- amount of additional training;

- amount of conventional therapy;

- length of intervention;

- pre-intervention outcome level; and

- phase poststroke.

We will only perform meta-regression if we include more than 10 trials in the analysis (McKenzie 2019).

If one of these moderators has a significant influence $(P<0.05)$ on the variability of the effect size, we will include those moderators in a mixed-effect model to evaluate whether those moderators explain the heterogeneity of the effect size. To examine if a trial is an outlier, we will use funnel plots, influential case diagnostics, and analyse the internally and externally standardised residuals. If the externally standardised residuals of an RCT are higher than \pm 1.96 in absolute value (Viechtbauer 2010b), we will define that RCT as an outlier and will perform an analysis with and without the outliers.

\section{Sensitivity analysis}

We will perform a sensitivity analysis for risk of bias in our included studies to test the robustness of our results for our primary outcome. We will exclude all trials with a high risk of bias for the five domains: randomisation, concealed allocation, blinding of assessors and participants, intention-to-treat, and other sources of bias. Finally, we will conduct meta-analyses with and without trials for which we imputed the calculated SDs, when six trials or more are included in the meta-analysis (Dealing with missing data, Fu 2010).

\section{Summary of findings and assessment of the certainty of the evidence}

We will create one 'Summary of findings' table using the following outcomes: activities of daily living, trunk function, arm-hand function, standing balance, walking ability, and quality of life after stroke comparing any type of trunk training versus non-dosematched or dose-matched control therapy (Table 2). We will use the five GRADE considerations (study limitations, consistency of effect, imprecision, indirectness and publication bias) to assess the certainty of evidence as it relates to the studies that contribute data to the meta-analyses for the prespecified outcomes (Atkins 2004). We will use methods and recommendations described in the Cochrane Handbook for Systematic Reviews of Interventions (Higgins 2019b), using GRADEproGDT software (GRADEproGDT). We will justify all decisions to downgrade the quality of studies using footnotes, and we will make comments to aid the reader's understanding of the review where necessary.

\section{ACKNOWLEDGEMENTS}

We thank Joshua Cheyne for help in providing the search strategy. 


\section{REFERE N C E S}

\section{Additional references}

\section{Ada 2006}

Ada L, Dean C, Mackey F. Increasing the amount of physical activity undertaken after stroke. Physical Therapy Reviews 2006;11:91-100.

\section{Alhwoaimel 2018}

Alhwoaimel N, Turk R, Warner M, Verheyden G, Thijs L, Wee SK, et al. Do trunk exercises improve trunk and upper extremity performance, post stroke? A systematic review and metaanalysis. NeuroRehabilitation 2018;43(4):395-412. [DOI: 10.3233/ NRE-182446]

\section{An 2017}

An S-H, Park D-S. The effects of trunk exercise on mobility, balance and trunk control of stroke patients. Journal of Korean Academy of Rehabilitation Medicine 2017;12(1):25-33. [DOI: 10.13066/kspm.2017.12.1.25]

\section{Atkins 2004}

Atkins D, Best D, Briss PA, Eccles M, Falck-Ytter Y, Flottorp S, et al. GRADE Working Group. Grading quality of evidence and strength of recommendations. BMJ 2004;328(7454):1490-8.

\section{Bank 2016}

Bank J, Charles K, Morgan P. What is the effect of additional physiotherapy on sitting balance following stroke compared to standard physiotherapy treatment: a systematic review. Topics in Stroke Rehabilitation 2016;23(1):15-25. [DOI: 10.1179/1945511915Y.0000000005]

\section{Berg 1992}

Berg KO, Wood-Dauphinee SL, Williams JI, Maki B. Measuring balance in the elderly: validation of an instrument. Canadian Journal of Public Health 1992;83 Suppl 2:S7-11.

\section{Bernhardt 2017}

Bernhardt J, Hayward K, Kwakkel G, Ward N, Wolf S, Borschmann K, et al. Agreed definitions and a shared vision for new standards in stroke recovery research: the Stroke Recovery and Rehabilitation Roundtable Taskforce. International Journal of Stroke 2017;12(5):444-50. [DOI: 10.1177/1747493017711816]

\section{Bohannon 1992}

Bohannon RW. Lateral trunk flexion strength: impairment, measurement reliability and implications following unilateral brain lesion. International Journal of Rehabilitation Research 1992;15(3):249-51.

\section{Cabanas-Valdés 2013}

Cabanas-Valdés R, Cuchi GU, Bagur-Calafat C. Trunk training exercises approaches for improving trunk performance and functional sitting balance in patients with stroke: a systematic review. NeuroRehabilitation 2013;33(4):575-92. [DOI: 10.3233/ NRE-130996]

\section{Collen 1990}

Collen FM, Wade DT, Bradshaw CM. Mobility after stroke: reliability of measures of impairment and disability. International Disability Studies 1990;12(1):6-9.

\section{Collin 1988}

Collin C, Wade DT, Davies S, Horne V. The Barthel ADL Index: a reliability study. International Disability Studies 1988;10(2):61-3.

\section{Collin 1990}

Collin C Wade D. Assessing motor impairment after stroke: a pilot reliability study. Journal of Neurology, Neurosurgery and Psychiatry 1990;53:576-9.

\section{Covidence 2017 [Computer program]}

Veritas Health Innovation Covidence. Version accessed 10 January 2017. Melbourne, Australia: Veritas Health Innovation. Available at: covidence.org.

\section{Davis 1990}

Davis PM. Problems associated with the loss of selective trunk activity in hemiplegia. In: Right in the Middle. New York: Springer, 1990:31-65.

\section{Deeks 2019}

Deeks JJ, Higgins JP, Altman DG, editor(s). Chapter 10: Analysing data and undertaking meta-analyses. In: Higgins JP, Thomas J, Chandler J, Cumpston M, Li T, Page MJ, et al, editor(s). Cochrane Handbook for Systematic Reviews of Interventions version 6.0 (updated July 2019). Cochrane, 2019. Available from www.training.cochrane.org/handbook.

\section{Di Monaco 2010}

Di Monaco M, Trucco M, Di Monaco R, Tappero R, Cavanna A. The relationship between initial trunk control or postural balance and inpatient rehabilitation outcome after stroke: a prospective comparative study. Clinical Rehabilitation 2010;24:543-54. [DOI: 10.1177/0269215509353265]

\section{Dickstein 1999}

Dickstein R, Heffes Y, Laufer Y, Ben-Haim Z. Activation of selected trunk muscles during symmetric functional activities in poststroke hemiparetic and hemiplegic patients. Journal of Neurology, Neurosurgery and Psychiatry 1999;66:218-21.

\section{Duarte 2002}

Duarte E, Marco E, Muniesa JM, Belmonte R, Diaz P, Tejero M, et al. Trunk control test as a functional predictor in stroke patients. Journal of Rehabilitation Medicine 2002;34(6):267-72. [DOI: 10.1080/165019702760390356]

\section{Duncan 1990}

Duncan PW, Weiner DK, Chandler J, Studenski S. Functional reach: a new clinical measure of balance. Journals of Gerontology 1990;45(6):M192-7. [DOI: 10.1093/ geronj/45.6.M192] 


\section{Duncan 1999}

Duncan PW, Wallace D, Lai SM, Johnson D, Embretson S, Laster LJ. The Stroke Impact Scale version 2.0. Evaluation of reliability, validity, and sensitivity to change. Stroke 1999;30(10):2131-40.

\section{Fu 2010}

Fu R, Gartlehner G, Grant M, Shamliyan T, Sedrakyan A, Wilt T, et al. Conducting quantitative synthesis when comparing medical interventions: AHRQ and the effective health care program. In: Methods Guide for Effectiveness and Comparative Effectiveness Reviews. Rockville: Agency for Healthcare Research and Quality, 2010:1-18. [http://effectivehealthcare.ahrq.gov/.]

\section{Fugl-Meyer 1975}

Fugl-Meyer AR, Jääskö L, Leyman I, Olsson S, Steglind S. The post-stroke hemiplegic patient: 1 . a method for evaluation of physical performance. Scandinavian Journal of Rehabilitation Medicine 1975;7(1):13-31.

\section{Fujino 2015}

Fujino Y, Amimoto K, Fukata K, Ishihara S, Makita S, Takahashi H. Does training sitting balance on a platform tilted $10^{\circ}$ to the weak side improve trunk control in the acute phase after stroke? A randomized, controlled trial. Topics in Stroke Rehabilitation 2015;23(1):43-9. [DOI: 10.1179/1945511915Y.0000000010]

\section{GBD 2019}

GBD 2016 Stroke Collaborators. Global, regional, and national burden of stroke, 1990-2016: a systematic analysis for the Global Burden of Disease Study. Lancet Neurology 2019;18(5):439-58.

\section{GRADEproGDT [Computer program]}

McMaster University (developed by Evidence Prime) GRADEpro GDT. Version accessed 6 July 2020. Hamilton (ON): McMaster University (developed by Evidence Prime). Available at gradepro.org.

\section{Harley 2006}

Harley C, Boyd JE, Cockburn J, Collin C, Haggard P, Wann JP, et al. Disruption of sitting balance after stroke: influence of spoken output. Journal of Neurology, Neurosurgery and Psychiatry 2006;77:674-6. [DOI: 10.1136/jnnp.2005.074138]

\section{Higgins 2003}

Higgins JP, Thompson SG, Deeks JJ, Altman DG. Measuring inconsistency in meta-analyses. BMJ 2003;327:557-60. [DOI: 10.1136/bmj.327.7414.557]

\section{Higgins 2011}

Higgins JP, Green S, editor(s). Cochrane Handbook for Systematic Reviews of Interventions Version 5.1.0 (updated March 2011). The Cochrane Collaboration, 2011. Available from handbook.cochrane.org.

\section{Higgins 2019a}

Higgins JP, Li T, Deeks JJ. Chapter 6: Choosing effect measures and computing estimates of effect. In: Higgins JP, Thomas J, Chandler J, Cumpston M, Li T, Page MJ, et al, editor(s). Cochrane Handbook for Systematic Reviews of Interventions version 6.0 (updated July 2019). Cochrane, 2019. Available from www.training.cochrane.org/handbook.

\section{Higgins 2019b}

Higgins JP, Thomas J, Chandler J, Cumpston M, Li T, Page MJ, et al, editor(s). Cochrane Handbook for Systematic Reviews of Interventions version 6.0 (updated July 2019). Cochrane, 2019. Available from www.training.cochrane.org/handbook.

\section{Higgins 2019c}

Higgins JP, Eldridge S, Li T, editors). Chapter 23: Including variants on randomized trials. In: Higgins JP, Thomas J, Chandler J, Cumpston M, Li T, Page MJ, et al, editor(s). Cochrane Handbook for Systematic Reviews of Interventions version 6.0 (updated July 2019). Cochrane, 2019. Available from www.training.cochrane.org/handbook. [www.training.cochrane.org/handbook.]

\section{Hoffmann 2014}

Hoffmann TC, Glasziou PP, Boutron I, Milne R, Perera R, Moher D. Better reporting of interventions: template for intervention description and replication (TIDieR) checklist and guide. BMJ 2014;348:g1687. [DOI: 10.1136/bmj.g1687]

\section{Holden 1984}

Holden MK, Gill KM, Magliozzi MR, Nathan J, PiehlBaker L. Clinical gait assessment in the neurologically impaired: reliability and meaningfulness. Physical Therapy 1984;64(1):35-40

\section{Houglum 2012}

Houglum P, Bertoti D. Brunnstrom's Clinical Kinesiology. Sixth edition. Philadelphia: FA Davis Company, 2012. [ISBN-13: 978-0-8036-2352-1]

\section{Hsieh 2002}

Hsieh C-L, Sheu C-F, Hsueh I-P, Wang C-H. Trunk control as an early predictor of comprehensive activities of daily living function in stroke patients. Stroke 2002;33:2626-30. [DOI: 10.1161/01.STR.0000033930.05931.93]

\section{Jung 2016}

Jung K-S, Jung J-H, In T-S, Cho, H-Y. Effects of weight-shifting exercise combined with transcutaneous electrical nerve stimulation on muscle activity and trunk control in patients with stroke. Occupational Therapy International 2016;23:436-43. [DOI: 10.1002/oti.1446]

\section{Karthikbabu 2011}

Karthikbabu S, Rao B, Manikandan N, Solomon J, Chakrapani M, Nayak A. Role of trunk rehabilitation on trunk control, balance and gait in patients with chronic stroke: a pre-post design. Neuroscience \& Medicine 2011;2:61-7. [DOI: 10.4236/ nm.2011.22009]

\section{Karthikbabu 2011a}

Karthikbabu S, Nayak A, Vijayakumar K, Misri ZK, Suresh BV, Ganesan S, et al. Comparison of physio ball and plinth trunk exercises regimens on trunk control and functional balance in patients with acute stroke: a pilot randomized 
controlled trial. Clinical Rehabilitation 2011;25:709-19. [DOI: 10.1177/0269215510397393]

\section{Keith 1987}

Keith R, Granger C, Hamilton B, Sherwin F. The Functional Independence Measure: a new tool for rehabilitation. Advances in Clinical Rehabilitation 1987;1:6-18.

\section{Ko 2016}

Ko EJ, Chun MH, Kim DY, Yi JH, Kim W, Hong J. The additive effects of core muscle strengthening and trunk NMES on trunk balance in stroke patients. Annals of Physical and Rehabilitation Medicine 2016;40(1):142-51. [DOI: 10.5535/arm.2016.40.1.142]

\section{Lee 2015}

Lee KB, Lim SH, Kim KH, Kim KJ, Kim YR, Chang WN, et al. Sixmonth functional recovery of stroke patients: a multi-timepoint study. International Journal of Rehabilitation Research 2015;38(2):173-80. [DOI: 10.1097/MRR.0000000000000108]

\section{Lee 2018}

Lee K, Cho J-E, Hwang D-Y, Lee W. Decreased respiratory muscle function is associated with impaired trunk balance among chronic stroke patients: a cross-sectional study. Tohoku Journal of Experimental Medicine 2018;245:79-88. [DOI: 10.1620/ tjem.245.79]

\section{Lefebvre 2019}

Lefebvre C, Glanville J, Briscoe S, Littlewood A, Marshall C, Metzendorf M-I, et al. Technical Supplement to Chapter 4: Searching for and selecting studies. In: Higgins JP, Thomas J, Chandler J, Cumpston M, Li T, Page MJ, et al, editor(s). Cochrane Handbook for Systematic Reviews of Interventions version 6.0 (updated July 2019). Cochrane, 2019. Available from www.training.cochrane.org/handbook.

\section{Liberati 2009}

Liberati A, Altman DG, Tetzlaff J, Mulrow C, Gøtzsche PC, loannidis JP, et al. The PRISMA Statement for reporting systematic reviews and meta-analyses of studies that evaluate health care interventions: explanation and elaboration. BMJ 2009;6(7):e1000100. [DOI: 10.1371/journal.pmed.1000100]

\section{Lyle 1981}

Lyle RC. A performance test for assessment of upper limb function in physical rehabilitation treatment and research. International Journal of Rehabilitation Research 1981;4(4):483-92.

\section{Mahoney 1965}

Mahoney FI, Barthel DW. Functional evaluation: the Barthel Index. Maryland State Medical Journal 1965;14:61-5.

\section{Mathias 1986}

Mathias S, Nayak US, Isaacs B. Balance in elderly patients: the "get-up and go" test. Archives of Physical Medicine and Rehabilitation 1986;67(6):387-9.

\section{McKenzie 2019}

McKenzie JE, Brennan SE, Ryan RE, Thomson HJ, Johnston RV. Chapter 9: Summarizing study characteristics and preparing for synthesis. In: Higgins JP, Thomas J, Chandler J, Cumpston M, Li T, Page MJ, et al, editor(s). Cochrane Handbook for Systematic Reviews of Interventions version 6.0 (updated July 2019). Cochrane, 2019. Available from www.training.cochrane.org/ handbook. [www.training.cochrane.org/handbook.]

\section{Messier 2004}

Messier S, Bourbonnais D, Desrosiers J, Roy Y. Dynamic analysis of trunk flexion after stroke. Archives of Physical Medicine and Rehabilitation 2004;85:1619-24. [DOI: 10.1016/ j.apmr.2003.12.043]

\section{Moreland 1993}

Moreland J, Gowland C, Van Hullenaar S, Huijbregts M. Theoretical basis of the Chedoke-McMaster Stroke Assessment. Physiotherapy Canada 1993;45(4):231-8.

\section{Quintino 2018}

Quintino LF, Franco J, Gusmão AF, Silva PF, Faria CD. Trunk flexor and extensor muscle performance in chronic stroke patients: a case-control study. Brazilian Journal of Physical Therapy 2018;22(3):231-7. [DOI: 10.1016/j.bjpt.2017.12.002]

\section{$\mathbf{R}$ [Computer program]}

R Foundation for Statistical Computing R: A language and environment for statistical computing. Version 3.4.2. Vienna, Austria: R Foundation for Statistical Computing, 2017. Available at www.R-project.org.

\section{RevMan Web [Computer program]}

The Cochrane Collaboration Review Manager Web (RevMan Web). The Cochrane Collaboration, 2019. Available at: revman.cochrane.org.

\section{Ryerson 2008}

Ryerson S, Byl N, Brown D, Wong R, Hidler J. Altered Trunk Position Sense and Its Relation to Balance Functions in People Post-Stroke. Journal of Neurologic Physical Therapy 2008;32(1):14-20.

\section{Santos 2019}

Santos R, Dall'Alba S, Forgiarini S, Rossato D, Dias A, Forgiarini L. Relationship between pulmonary function, functional independence, and trunk control in patients with stroke. Arquivos de Neuro-Psiquiatria 2019;77(6):387-92. [DOI: 10.1590/0004-282×20190048]

\section{Silva 2015}

Silva P, Franco J, Gusmão A, Moura J, Teixeira-Salmela L, Faria C. Trunk strength is associated with sit-to-stand performance in both stroke and healthy subjects. European Journal of Physical and Rehabilitation Medicine 2015;51:717-24. [PMID: 25673183]

\section{Smith 1999}

Smith M, Baer G. Achievement of simple mobility milestones after stroke. Archives of Physical Medicine and Rehabilitation 1999;80:442-7.

\section{Sorinola 2014}

Sorinola IO, Powis I, White CM. Does additional exercise improve trunk function recovery in stroke patients? A meta- 
analysis. NeuroRehabilitation 2014;35:205-13. [DOI: 10.3233/ NRE-141123]

\section{Souza 2019}

Souza DC, de Sales Santos M, da Silva Ribeiro NM, Maldonado IL. Inpatient trunk exercises after recent stroke: an update meta-analysis of randomized controlled trials. NeuroRehabilitation 2019;44(3):369-77. [DOI: 10.3233/ NRE-182585]

\section{Sterne 2005}

Sterne JA, Egger M. Regression methods to detect publication and other bias in meta-analysis. In: John Wiley \& Sons.

Chichester: HR Rothstein, AJ Sutton, M Borenstein, 2005:99-110.

\section{Tanaka 1998}

Tanaka S, Hachisuka K, Ogata H. Muscle strength of trunk flexion-extension in post-stroke hemiplegic patients. American Journal of Physical Medicine \& Rehabilitation 1998;77(4):288-90.

\section{Tinetti 1986}

Tinetti ME, Williams TF, Mayewski R. Fall Risk Index for elderly patients based on number of chronic disabilities. American Journal of Medicine 1986;80:429-34.

\section{Van Criekinge 2019}

Van Criekinge T, Truijen S, Schröder J, Maebe Z, Blanckaert K, van der Waal C, et al. The effectiveness of trunk training on trunk control, sitting and standing balance and mobility post-stroke: a systematic review and meta-analysis. Clinical Rehabilitation 2019;33(6):992-1002.

\section{Verheyden 2004}

Verheyden G, Mertin J, Preger R, Kiekens C, Weerdt WD. The Trunk Impairment Scale: a new tool to measure motor impairment of the trunk after stroke. Clinical Rehabilitation 2004;18:326-33.

\section{Verheyden 2006}

Verheyden G, Vereeck L, Truijen S, Troch M, Herregodts I, Lafosse $C$, et al. Trunk performance after stroke and relationship with balance, gait and functional ability. Clinical Rehabilitation 2006;20:451-8.

\section{Verheyden 2007}

Verheyden G, Nieuwboer A, De Wit L, Feys H, Schuback B, Baert I, et al. Trunk performance after stroke: an eye catching predictor of functional outcome. Journal of Neurology, Neurosurgery and Psychiatry 2007;78:694-8.

\section{Viechtbauer 2010a}

Viechtbauer W. Conducting meta-analyses in R with the metafor package. Journal of Statistical Software 36;3:1-48. [jstatsoft.org/ $\mathrm{v} 36 / \mathrm{i} 03 /]$

\section{Viechtbauer 2010b}

Viechtbauer W, Cheung MW-L. Outlier and influence diagnostics for meta-analysis. Research Synthesis Methods 2010;1:112-25.

\section{Wan 2014}

Wan X, Wang W, Liu J, Tong T. Estimating the sample mean and standard deviation from the sample size, median, range and/or interquartile range. BMC Medical Research Methodology 2014;14(135):1-13. [DOI: 10.1186/1471-2288-14-135]

\section{Wee 2015}

Wee KS, Hughes A-M, Warner M, Brown S, Cranny A, Mazomenos $\mathrm{E}$, et al. Effect of trunk support on upper extremity function in people with chronic stroke and people who are healthy. Physical Therapy 2015;95(8):1163-71.

\section{WHO 2001}

World Health Organization. International classification of functioning, disability and health: ICF. World Health Organization. who.int/iris/handle/10665/42407 2001.

\section{Wood-Dauphinee 1988}

Wood-Dauphinee SL, Opzoomer MA, Williams JI, Marchand B, Spitzer WO. Assessment of global function: The reintegration to normal living index. Archives of Physical Medicine and Rehabilitation 1988;69(8):583-90.

\section{Yoo 2010}

Yoo SD, Jeong YS, Kim DH, Lee M-A, Noh SG, Shin YW, et al. The efficacy of core strengthening on the trunk balance in patients with subacute stroke. Journal of Korean Academy of Rehabilitation Medicine 2010;34:677-82.

\section{ADDITIONAL TABLES}

\section{Table 1. Overview of published reviews on trunk training}

\begin{tabular}{llllll}
\hline Review & Cabanas-Valdés 2013 & Sorinola 2014 & $\begin{array}{l}\text { Alhwoaimel } \\
\mathbf{2 0 1 8}\end{array}$ & $\begin{array}{l}\text { Van Criekinge } \\
\mathbf{2 0 1 9}\end{array}$ & Souza 2019 \\
\hline Aim & $\begin{array}{l}\text { To evaluate the effec- } \\
\text { tiveness of trunk train- } \\
\text { ing exercises on trunk } \\
\text { performance, sitting } \\
\text { balance, standing bal- } \\
\text { ance, and gait. }\end{array}$ & $\begin{array}{l}\text { To establish the effica- } \\
\text { cy of additional trunk } \\
\text { exercise on trunk func- } \\
\text { tion, balance, walking } \\
\text { ability, and functional } \\
\text { independence early af- } \\
\text { ter stroke. }\end{array}$ & $\begin{array}{l}\text { To evaluate the } \\
\text { effects of trunk } \\
\text { training on trunk } \\
\text { control and up- } \\
\text { per extremity } \\
\text { function. }\end{array}$ & $\begin{array}{l}\text { To study ef- } \\
\text { fectiveness of } \\
\text { trunk training } \\
\text { on standing } \\
\text { balance, and } \\
\text { mobility. }\end{array}$ & $\begin{array}{l}\text { To assess the im- } \\
\text { pact of the addition } \\
\text { of specific inpatient } \\
\text { trunk training in the } \\
\text { first 3 months after } \\
\text { stroke. }\end{array}$ \\
& RCTs & RCTs & RCTs & RCTs & RCTs \\
\hline Type of studies & RCTs & & \\
\hline
\end{tabular}


Table 1. Overview of published reviews on trunk training (Continued)

\begin{tabular}{|c|c|c|c|}
\hline Clear distinc- & No & No & No \\
\hline $\begin{array}{l}\text { which experi- } \\
\text { mental group } \\
\text { receives addi- } \\
\text { tional therapy }\end{array}$ & $\begin{array}{l}\text { Therapy intervention } \\
\text { in control group is a } \\
\text { combination of no } \\
\text { therapy, non-dose- } \\
\text { matched therapy and } \\
\text { dose-matched therapy. }\end{array}$ & $\begin{array}{l}\text { Therapy intervention } \\
\text { in control group is a } \\
\text { combination of no } \\
\text { therapy, non-dose- } \\
\text { matched therapy and } \\
\text { dose-matched therapy. }\end{array}$ & $\begin{array}{l}\text { Therapy inter- } \\
\text { vention in con- } \\
\text { trol group is a } \\
\text { combination of } \\
\text { no therapy, non- } \\
\text { dose-matched } \\
\text { therapy and } \\
\text { dose-matched } \\
\text { therapy. }\end{array}$ \\
\hline
\end{tabular}

No

Therapy intervention in control group is a combination of no therapy, non-dosematched therapy and dosematched thera-

py.

\begin{tabular}{|c|c|c|c|c|c|}
\hline $\begin{array}{l}\text { Number of stud- } \\
\text { ies included }\end{array}$ & $\begin{array}{l}11 \\
\text { studies } \\
\text { (317 participants) }\end{array}$ & $\begin{array}{l}6 \text { studies } \\
\text { (155 participants) }\end{array}$ & $\begin{array}{l}17 \text { studies } \\
\text { (599 partici- } \\
\text { pants) }\end{array}$ & $\begin{array}{l}22 \text { studies } \\
\text { (788 partici- } \\
\text { pants) }\end{array}$ & $\begin{array}{l}9 \text { studies } \\
\text { (358 participants) }\end{array}$ \\
\hline $\begin{array}{l}\text { Evaluation of } \\
\text { quality of evi- } \\
\text { dence }\end{array}$ & PEDro score & PEDro score & PEDro score & PEDro score & PEDro score \\
\hline \multirow{2}{*}{$\begin{array}{l}\text { Evaluation of } \\
\text { risk of bias }\end{array}$} & No & Yes & Yes & No & Yes \\
\hline & & $\begin{array}{l}\text { Cochrane 'Risk of bias' } \\
\text { tool }\end{array}$ & $\begin{array}{l}\text { Cochrane 'Risk of } \\
\text { bias' tool }\end{array}$ & & $\begin{array}{l}\text { Cochrane 'Risk of } \\
\text { bias' tool }\end{array}$ \\
\hline \multirow{2}{*}{$\begin{array}{l}\text { Performed } \\
\text { meta-analysis }\end{array}$} & No & Yes & Yes & Yes & Yes \\
\hline & Narrative review & & & & $\begin{array}{l}\text { Performed on limit- } \\
\text { ed number of stud- } \\
\text { ies, with same out- } \\
\text { come measure }\end{array}$ \\
\hline \multirow{2}{*}{$\begin{array}{l}\text { Distinction be- } \\
\text { tween type or } \\
\text { therapy }\end{array}$} & Yes & No & No & No & No \\
\hline & $\begin{array}{l}\text { Sitting reaching train- } \\
\text { ing and selective trunk } \\
\text { training }\end{array}$ & & & & \\
\hline Last search date & November 2012 & July 2012 & February 2017 & January 2019 & December 2017 \\
\hline
\end{tabular}

\section{Evaluated outcomes}

\begin{tabular}{|c|c|c|c|c|c|}
\hline Trunk function & Yes & Yes & Yes & Yes & Yes \\
\hline & 10 RCTs & 6 RCTs & 17 RCTs & $20 \mathrm{RCTs}$ & 8 RCTs \\
\hline \multirow{2}{*}{$\begin{array}{l}\text { Standing bal- } \\
\text { ance }\end{array}$} & Yes & Yes & No & Yes & Yes \\
\hline & 6 RCTs & 2 RCTs & & 6 RCTs & 4 RCTs \\
\hline \multirow[t]{2}{*}{ Gait } & Yes & Yes & No & Yes & No \\
\hline & 5 RCTs & 3 RCTs & & 8 RCTs & \\
\hline \multirow{2}{*}{$\begin{array}{l}\text { Functional per- } \\
\text { formance }\end{array}$} & No & Yes & No & No & No \\
\hline & & 2 RCTs & & & \\
\hline
\end{tabular}


Table 1. Overview of published reviews on trunk training (Continued)

\begin{tabular}{|c|c|c|c|c|c|}
\hline $\begin{array}{l}\text { Upper limb out- } \\
\text { comes }\end{array}$ & No & No & $\begin{array}{l}\text { Yes } \\
\text { No studies in- } \\
\text { cluded }\end{array}$ & No & No \\
\hline $\begin{array}{l}\text { Activities of dai- } \\
\text { ly living }\end{array}$ & No & No & No & No & No \\
\hline Quality of life & $\begin{array}{l}\text { Yes } \\
1 \mathrm{RCT}\end{array}$ & No & No & No & No \\
\hline Adverse events & No & No & No & No & No \\
\hline Other outcomes & No & No & No & No & No \\
\hline Conclusion & $\begin{array}{l}\text { Moderate evidence to } \\
\text { improve trunk perfor- } \\
\text { mance and quality of } \\
\text { life. } \\
\text { Trials were inconclu- } \\
\text { sive about outcome on } \\
\text { gait and balance. }\end{array}$ & $\begin{array}{l}\text { Moderate, non-signif- } \\
\text { icant effect on trunk } \\
\text { function, } \\
\text { large effects on stand- } \\
\text { ing balance, small, } \\
\text { non-significant effect } \\
\text { on functional indepen- } \\
\text { dence }\end{array}$ & $\begin{array}{l}\text { Large significant } \\
\text { effect on trunk } \\
\text { performance }\end{array}$ & $\begin{array}{l}\text { Large signifi- } \\
\text { cant effect on } \\
\text { trunk control, } \\
\text { standing bal- } \\
\text { ance and mo- } \\
\text { bility }\end{array}$ & $\begin{array}{l}\text { Significant im- } \\
\text { provement in trunk } \\
\text { control and balance }\end{array}$ \\
\hline
\end{tabular}

$\mathrm{RCT}$ : randomised controlled trial

Table 2. Summary of findings

Trunk training compared with control intervention for people after stroke

Patient or population: participants after stroke

Settings: hospital, clinic, inpatient rehabilitation centre

Intervention: all types of trunk training

Comparison: non-dose-matched or dose-matched therapy

\begin{tabular}{|c|c|c|c|c|c|}
\hline Outcomes & $\begin{array}{l}\text { Out- } \\
\text { come } \\
\text { mea- } \\
\text { sures }\end{array}$ & $\begin{array}{l}\text { Relative } \\
\text { effect } \\
(95 \% \mathrm{Cl})^{\star}\end{array}$ & $\begin{array}{l}\text { No of Par- } \\
\text { ticipants } \\
\text { (studies) }\end{array}$ & $\begin{array}{l}\text { Certainty of } \\
\text { the evidence } \\
\text { (GRADE) }\end{array}$ & $\begin{array}{l}\text { Com- } \\
\text { ments }\end{array}$ \\
\hline
\end{tabular}

Activities of daily living

Trunk function

Arm-hand activity

Standing balance

Walking ability

Quality of life after stroke 
Table 2. Summary of findings (Continued)

*The risk in the intervention group (and its 95\% confidence interval) is based on the assumed risk in the comparison group and the relative effect of the intervention (and its $95 \% \mathrm{Cl}$ ).

Cl: confidence interval

GRADE Working Group grades of evidence

High certainty: Further research is very unlikely to change our confidence in the estimate of effect.

Moderate certainty: Further research is likely to have an important impact on our confidence in the estimate of effect and may change the estimate.

Low certainty: Further research is very likely to have an important impact on our confidence in the estimate of effect and is likely to change the estimate.

Very low certainty: We are very uncertain about the estimate.

\section{APPENDICES}

\section{Appendix 1. MEDLINE Ovid search strategy}

1. cerebrovascular disorders/ or basal ganglia cerebrovascular disease/ or exp brain ischemia/ or exp carotid artery diseases/ or exp cerebral small vessel diseases/ or exp intracranial arterial diseases/ or exp "intracranial embolism and thrombosis"/ or exp intracranial hemorrhages/ or stroke/ or exp brain infarction/ or stroke, lacunar/ or vasospasm, intracranial/ or vertebral artery dissection/

2. (stroke\$ or poststroke or apoplex\$ or cerebral vasc\$ or brain vasc\$ or cerebrovasc\$ or cva $\$$ or SAH).tw.

3. ( (brain $\$$ or cerebr $\$$ or cerebell\$ or vertebrobasil\$ or hemispher $\$$ or intracran $\$$ or intracerebral or infratentorial or supratentorial or middle cerebral artery or MCA\$ or anterior circulation or posterior circulation or basilar artery or vertebral artery or space-occupying) adj5 (isch? emi\$ or infarct\$ or thrombo\$ or emboli\$ or occlus\$ or hypoxi\$)).tw.

4. ((brain\$ or cerebr\$ or cerebell\$ or intracerebral or intracran\$ or parenchymal or intraparenchymal or intraventricular or infratentorial or supratentorial or basal gangli\$ or putaminal or putamen or posterior fossa or hemispher\$ or subarachnoid) adj5 (h?emorrhag\$ or h? ematoma\$ or bleed\$)).tw.

5. hemiplegia/ or exp paresis/

6. (hemipleg\$ or hemipar\$ or paresis or paraparesis or paretic).tw.

7. or/1-6

8. exp torso/

9. exp abdominal muscles/ or exp back muscles/ or pectoralis muscles/ or exp respiratory muscles/

10. (trunk or truncal or thorax or thoracic or torso or diaphragm $\$$ or intercostal or pectoral\$ or ((rib or chest) adj3 (cavity or cage)) or pelvi $\$$ or abdom $\$$ or perine $\$$ or peritonial or (core adj3 stabil\$)).tw.

11. (back or erector spinae or spinal erector\$ or sacrospinal\$ or latissimus dorsi or levator scapulae or multifidus or paraspinal or trapezius).tw.

12. or/8-11

13. exp physical therapy modalities/

14. occupational therapy/ or physical therapy specialty/

15. "physical and rehabilitation medicine"/ or rehabilitation/ or exp neurological rehabilitation/ or telerehabilitation/

16. movement/ or locomotion/ or exp running/ or swimming/ or walking/or dependent ambulation/ or exp gait/ or motor activity/ or exp exercise/

17. exp sports/

18. (exercis\$ or train $\$$ or condition $\$$ or strengthen $\$$ or rehab\$ or stabili\$).tw.

19. or $/ 13-18$

20. randomized controlled trial.pt.

21. controlled clinical trial.pt.

22. randomized.ab.

23. placebo.ab.

24. randomly.ab.

25. trial.ab.

26. groups.ab.

27. or $/ 20-26$

28. 7 and 12 and 19 and 27

\section{HISTORY}

Protocol first published: Issue 9, 2020 


\section{CONTRIBUTIONS OF AUTHORS}

Thijs L: wrote the protocol and designed search strategies.

Denissen S: provided general advice on the protocol.

Mehrholz J: provided general advice on the protocol.

Bernhard E: provided general advice on the protocol.

Lemmens R: provided general advice on the protocol.

Verheyden G: wrote the protocol.

\section{DECLARATIONS OF INTEREST}

Thijs L: none known

Denissen S: none known

Mehrholz J: none known

Bernhard E: none known

Lemmens R: none known

Verheyden G: none known

\section{SOURCES OF SUPPORT}

\section{Internal sources}

- No sources of support supplied

\section{External sources}

- KU Leuven internal funding (RUN-17-00175), Belgium

- EU Horizon 2020 Eurostars funding (E! 11323), Other

- Baekeland grant ( HBC.2019.2579), Belgium 